\title{
Gelsemine alleviates both neuropathic pain and sleep disturbance in partial sciatic nerve ligation mice
}

\author{
Yu-er WU ${ }^{1,2, \#}$, Ya-dong $\mathrm{LI}^{2, \#}$, Yan-jia LUO ${ }^{2}$, Tian-xiao WANG ${ }^{2}$, Hui-jing WANG ${ }^{2, *}$, Shuo-nan $\mathrm{CHEN}^{2}$, Wei-min QU ${ }^{2,3}$, Zhi-li \\ HUANG $^{1,2,3, *}$
}

\author{
${ }^{1}$ State Key Laboratory of Medical Neurobiology, School of Basic Medical Sciences, Fudan University, Shanghai 200032, China; \\ ${ }^{2}$ Department of Pharmacology and Shanghai Key Laboratory of Bioactive Small Molecules, School of Basic Medical Sciences, Fudan \\ University, Shanghai 200032, China; ${ }^{3}$ Institutes of Brain Science and Collaborative Innovation Center for Brain Science, Fudan \\ University, Shanghai 200032, China
}

\begin{abstract}
Aim: Gelsemine, an alkaloid from the Chinese herb Gelsemium elegans (Gardn \& Champ) Benth, is effective in mitigating chronic pain in rats. In the present study we investigated whether the alkaloid improved sleep disturbance, the most common comorbid symptoms of chronic pain, in a mouse model of neuropathic pain.

Methods: Mice were subjected to partial sciatic nerve ligation (PSNL). After the mice were injected with gelsemine or pregabalin (the positive control) intraperitoneally, mechanical allodynia and thermal hyperalgesia were assessed, and electroencephalogram (EEG)/ electromyogram (EMG) recording was performed. Motor performance of the mice was assessed using rota-rod test. c-Fos expression in the brain was analyzed with immunohistochemical staining.

Results: In PSNL mice, gelsemine ( 2 and $4 \mathrm{mg} / \mathrm{kg}$ ) increased the mechanical threshold for $4 \mathrm{~h}$ and prolonged the thermal latencies for $3 \mathrm{~h}$. Furthermore, gelsemine (4 mg/kg, administered at 6:30 AM) increased non-rapid eye movement (non-REM, NREM) sleep, decreased wakefulness, but did not affect REM sleep during the first $3 \mathrm{~h}$ in PSNL mice. Sleep architecture analysis showed that gelsemine decreased the mean duration of wakefulness and increased the total number of episodes of NREM sleep during the first 3 $\mathrm{h}$ after the dosing. Gelsemine (4 $\mathrm{mg} / \mathrm{kg}$ ) did not impair motor coordination in PSNL mice. Immunohistochemical study showed that PSNL increased c-Fos expression in the neurons of the anterior cingulate cortex, and gelsemine (4 mg/kg) decreased c-Fos expression by 58\%. Gelsemine (4 mg/kg, administered at either 6:30 AM or 8:30 PM) did not produce hypnotic effect in normal mice. Pregabalin produced similar antinociceptive and hypnotic effects, but impaired motor coordination in PSNL mice.

Conclusion: Gelsemine is an effective agent for treatment of both neuropathic pain and sleep disturbance in PSNL mice; anterior cingulate cortex might play a role in the hypnotic effects of gelsemine.
\end{abstract}

Keywords: anterior cingulate cortex; c-Fos; electroencephalogram; gelsemine; Gelsemium; neuropathic pain; pregabalin; sleep disturbance

Acta Pharmacologica Sinica (2015) 36: 1308-1317; doi: 10.1038/aps.2015.86; published online 21 Sep 2015

\section{Introduction}

Neuropathic pain, defined as pain resulting from lesions or diseases of the sensory transmission pathways in the peripheral or central nervous system, is the most difficult type of chronic pain to manage in the pain clinic ${ }^{[1,2]}$. Recently, both clinical and experimental studies have provided evidence linking sleep disturbance to neuropathic pain ${ }^{[3,4]}$. Specifically,

\footnotetext{
\#These authors contributed equally to this article.

* To whom correspondence should be addressed.

E-mail huangzl@fudan.edu.cn (Zhi-li HUANG); hjwang2@fudan.edu.cn (Hui-jing WANG)

Received 2015-05-05 Accepted 2015-06-07
}

more than $70 \%$ of patients with diverse chronic pain conditions, including neuropathic pain, complain of recurring sleep disturbances such as an increased sleep latency, increased wakefulness after sleep onset, and reduced slow wave sleep and shifts in sleep stages ${ }^{[5-7]}$. This suggests that an effective agent that exerts both analgesic and hypnotic effects would provide multiple benefits in patients with sleep disturbance and neuropathic pain ${ }^{[8,9]}$. However, there is no ideal therapy that not only reduces pain, but also improves sleep without an increased risk of side effects. Antidepressants, which are considered to be first-line drugs for the treatment of neuropathic pain, only alleviate pain but do not ameliorate sleep disturbance $^{[9]}$. Therefore, new agents that exert both analgesic and 
hypnotic effects and possess more acceptable adverse effects should be explored.

Gelsemine (for chemical structure see Figure 1) is an active principle and major alkaloid from the Gelsemium elegans (Gardn \& Champ) Benth, one of Gelsemium species. Gelsemium is a small genus of flowering plant from the Gelsemiaceae family, comprising five species and three of them are particularly popular: Gelsemium elegans (Gardn \& Champ) Benth, native to East Asia, particularly in China; Gelsemium sempervirens (L.) J.St.Hil., commonly known as yellow jasmine, native to southwest United States; and Gelsemium rankinii Small, native to North America $^{[10,11]}$. Gelsemium elegans, known as Gou Wen or Duan Chang Cao in China, is first recorded in The Shennong Emperor's Classic of Materia Medica (Shen-nong Ben-cao Jing), the classic Chinese herbal medicine. Gelsemium elegans has been used to treat skin ulcers, headaches, neuropathic pain and cancer pain for many years. More than 190 compounds have been found in Gelsemium, but the primary active components are alkaloids. The alkaloids extracted from the plant have been found possess various biological effects ${ }^{[12-17]}$. Recently, studies demonstrated that gelsemine was effective at mitigating chronic pain in rats without apparent side effects, and was an appropriate agent to treat neuropathic pain ${ }^{[18]}$. However, the actions exerted by gelsemine on sleep disturbance, the most common comorbid symptoms of chronic pain, have received little attention.

In the present study, we evaluated the effects of gelsemine on sleep disturbance in a mouse partial sciatic nerve ligation (PSNL) model by recording electroencephalogram (EEG) and electromyogram (EMG). In addition, the anterior cingulate cortex (ACC) is a key cortical region for pain perception and unpleasantness ${ }^{[19,20]}$. Recent studies suggested that the ACC plays an important role in sleep disorders caused by pain ${ }^{[21,22]}$. We tested the hypothesis that ACC might be involved in the effect of gelsemine on sleep in PSNL mice. Here we used Fos expression as a marker of neuronal activation to quantify the responses of ACC neurons in PSNL mice after gelsemine administration.

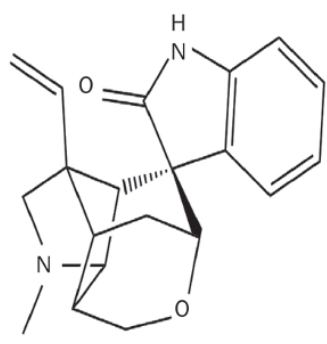

Figure 1. The chemical structure of gelsemine.

\section{Materials and methods} Animals

Male C57BL/6J mice weighing 20-26 g (11-13 weeks old) were obtained from the Laboratory Animal Center, Chinese Academy of Sciences (Shanghai, China). The animals were housed individually at an ambient temperature of $22 \pm 0.5^{\circ} \mathrm{C}$ with a relative humidity of $60 \% \pm 2 \%$, an automatically controlled $12 \mathrm{~h}$ light/dark cycle (lights on at 07:00 AM, illumination intensity $\approx 100$ lux), and free access to food and water. The Medical Experimental Animal Administrative Committee of Fudan University approved the experimental protocols. Every effort was made to minimize the number of animals used and any pain and discomfort experienced by the animals.

\section{Chemicals}

Gelsemine was purchased from the Chengdou Muster Biotechnology Co Ltd (lot number: MUST-14040104, Chengdu, China) and dissolved in sterile saline containing 1\% DMSO immediately before use. Pregabalin was purchased from Chongqing Sai Wei Pharmaceutical Co Ltd (Chongqing, China). Rabbit polyclonal anti-c-Fos antibodies were purchased from Millipore (Boston, MA, USA), and biotinylated goat anti-rabbit IgG, avidin-biotin-peroxidase, and 3,3-diamino-benzidine-tetrahydrochloride $(\mathrm{DAB})$ were obtained from Vector Laboratories (Burlingame, CA, USA).

\section{Neuropathic pain model}

The mice were anesthetized with $5 \%$ chloral hydrate [360 $\mathrm{mg} / \mathrm{kg}$, intraperitoneally (ip)]. A partial nerve injury model was produced by tying a tight 7-0 silk suture ligature around approximately one-third to half of the diameter of the sciatic nerve on the right side, as described previously ${ }^{[2,4,23]}$. In shamoperated mice, the nerve was exposed without ligation. PSNL or sham-operated mice were housed in separate cages to allow them to recover for $7 \mathrm{~d}$ and were then used in experiments simultaneously.

\section{Measuring mechanical allodynia and thermal hyperalgesia}

Mechanical allodynia was measured as the hind paw withdrawal response to von Frey hair stimulation according to the up-down method ${ }^{[24-27]}$. Animals were placed in a plastic cage with a framed metal mesh floor, and allowed to habituate for $60 \mathrm{~min}$ prior to testing. Von Frey hairs (bending force range of 0.1-8 g; North Coast Medical, Inc, San Jose, CA, USA) were applied perpendicular to the mid-plantar surface of the hind paw. Each hair was held for $\sim 3 \mathrm{~s}$, with a 10-min interval. A trial began with the application of the $1.4 \mathrm{~g}$ hair, and a positive response was defined as the withdrawal of the hind paw upon the stimulus. Whenever a positive response to a stimulus occurred the hair force was decreased, whereas hair force was increased whenever a negative response occurred. The testing consisted of five more stimuli after the first change in response occurred, and the $50 \%$ paw withdrawal threshold was calculated using the method described previously ${ }^{[28]}$.

Thermal hyperalgesia was assessed using a plantar test apparatus (IITC Inc/Life Science Instruments, Woodland Hills, CA, USA) as reported previously ${ }^{[29]}$ by measuring the hind paw withdrawal latency in response to radiant heat. Briefly, mice were placed into compartment enclosures on a glass sur- 
face. After a 60-min accommodation period, a mobile radiant heat source was focused on the hind paw and the paw withdrawal latencies were defined as the time taken by the mouse to remove its hind paw from the heat source. The intensity of the thermal stimulus was adjusted to achieve a mean baseline paw-withdrawal latency of $\sim 9-11 \mathrm{~s}$ in naive mice. A cutoff time of $20 \mathrm{~s}$ was imposed to prevent tissue damage in the absence of a response. The mean withdrawal latency for the right hind paw was determined as the mean of five separate trials performed at 5-min intervals to prevent thermal sensitization and behavioral disturbances.

\section{Polygraphic recordings and vigilance state analysis}

Under chloral hydrate anesthesia (360 mg/kg, ip), PSNL or sham-operated mice were implanted simultaneously with EEG and EMG electrodes for polysomnographic recordings. The implant consisted of two stainless steel screws (1 mm diameter) inserted through the skull over the cortex (anteroposterior, $+1.0 \mathrm{~mm}$; left-right, $-1.5 \mathrm{~mm}$ from the bregma or the lambda) according to the atlas of Franklin and Paxinos to serve as EEG electrodes. Two insulated stainless steel, Teflon-coated wires placed bilaterally into both trapezius muscles to function as EMG electrodes. All electrodes were attached to a micro connector and fixed to the skull using dental cement.

EEG and EMG recordings were performed using a slip ring that was designed to ensure that the behavioral movement of the mice would not be restricted ${ }^{[30,31]}$. After a 10-d recovery period, the mice were housed individually in transparent barrels and habituated to the recording cable for 3-4 $\mathrm{d}$ before the polygraphic recordings commenced. Sleep-wakefulness states were monitored for two consecutive periods of $24 \mathrm{~h}$, encompassing both baseline and the experimental day. Baseline recordings were taken in each animal for $24 \mathrm{~h}$ beginning at 07:00 AM, which served as the paired control for the same animal.

Cortical EEG and EMG signals were amplified, filtered (EEG, 0.5-30 Hz; EMG, 20-200 Hz), digitized at a sampling rate of $128 \mathrm{~Hz}$, and recorded using SleepSign software (Kissei Comtec, Nagano, Japan) as described previously ${ }^{[32-34]}$. When the recordings completed, vigilance was classified automatically offline under 4-s epochs into three stages [wakefulness, rapid eye movement (REM), and non-REM (NREM) sleep] using SleepSign according to standard criteria ${ }^{[32,35,36]}$. During the final step the defined sleep-wake stages were examined visually and corrected if necessary.

\section{Rota-rod test}

The rota-rod test was performed according to the method described by Kuribara et al ${ }^{[37]}$. The rota-rod apparatus (Med Associates, Georgia, VT, USA) consisted of a bar with a diameter of $3 \mathrm{~cm}$ that was subdivided into five compartments. The time taken to fall off the rota-rod was recorded as the latency (s). Before drug administration the mice were trained daily for $3 \mathrm{~d}$, and the rod was rotated at a constant speed of 16 revolutions per min. On the day of the test only mice that were able to stay balanced on the rotating rod for $180 \mathrm{~s}$ (cut-off time) were selected for testing. The results are expressed as the mean length of time the animals were able to remain on the rota-rod in each group.

\section{c-Fos immunohistochemistry}

Four groups of mice were used: PSNL+gelsemine $4 \mathrm{mg} / \mathrm{kg}$, PSNL+vehicle, sham+gelsemine $4 \mathrm{mg} / \mathrm{kg}$, and sham+vehicle. Each group was given either vehicle or gelsemine intraperitoneally at 6:30 AM. Two hours later, mechanical allodynia was measured in all animals that were then sacrificed for immunohistochemistry according to our previous description ${ }^{[38]}$. The animals were deeply anesthetized with an overdose of $5 \%$ chloral hydrate, and then perfused with phosphate-buffered saline (PBS) followed by ice-cold $4 \%$ paraformaldehyde (PFA) in $0.1 \mathrm{~mol} / \mathrm{L}$ phosphate buffer $(\mathrm{PB}, \mathrm{pH} 7.0)$ through the heart. The brains were then post-fixed in the same fixative at $4^{\circ} \mathrm{C}$ for 2-4 h. Subsequently, the brains were cryoprotected in $20 \%$ sucrose in PBS at $4{ }^{\circ} \mathrm{C}$ for $1 \mathrm{~d}$ followed by $30 \%$ sucrose in PBS for $2 \mathrm{~d}$. Thereafter, 30- $\mu \mathrm{m}$ frozen sections were cut in coronal planes using a freezing microtome (Jung Histocut, model820II, Leica, Nussloch, Germany). The sections were washed in PBS and then incubated in $0.6 \% \mathrm{H}_{2} \mathrm{O}_{2}$ for $30 \mathrm{~min}$. After being washed with PBS, the sections were blocked with antiserum solution 1 [10\% normal bovine serum albumin (BSA), $0.2 \%$ Triton X-100, and $0.4 \%$ sodium azide in $0.01 \mathrm{~mol} / \mathrm{L} \mathrm{PBS}$ at $\mathrm{pH}$ 7.2] for $30 \mathrm{~min}$. The sections were then incubated with c-Fos antibodies (rabbit polyclonal, ABE-457, Millipore, Boston, MA, USA) that had been diluted 1:10000 in antiserum solution 2 ( $1 \%$ normal BSA, $0.2 \%$ Triton $\mathrm{X}-100$, and $0.4 \%$ sodium azide in $0.01 \mathrm{~mol} / \mathrm{L}$ PBS at $\mathrm{pH} 7.2$ ) overnight at room temperature. On the second day the sections were washed in PBS and incubated with biotinylated goat anti-rabbit secondary antibodies (1:1000 in PBS) for $1 \mathrm{~h}$, followed by a 1:800 dilution of avidinbiotin-peroxidase for $1 \mathrm{~h}$ at $37^{\circ} \mathrm{C}$. The peroxidase reaction was visualized with $0.05 \%$ DAB in PBS and $0.01 \% \mathrm{H}_{2} \mathrm{O}_{2}$. After terminating the reaction by the addition of PBS-azide, the sections were mounted, dehydrated, and coverslipped. The sections were examined under bright-field illumination using a Leica DMLB2 microscope (Leica Microsystems, Wetzlar, Germany). Images were then captured using a Cool SNAP-Proof digital camera (SPOT RTKE Diagnostic Instruments, Sterling Heights, MI, USA). Brain slices for immunohistochemistry corresponded approximately to ACC (from $1.0 \mathrm{~mm}$ anterior to bregma to $0.5 \mathrm{~mm}$ anterior to bregma) according to the atlas of Franklin and Paxinos. The number of c-Fos-positive neurons was counted in two adjacent sections that contained ACC structures. A $0.2 \mathrm{~mm} \times 0.2 \mathrm{~mm}$ counting box was placed in the center of the ACC.

\section{Pharmacological treatments}

To study the antinociceptive effects of gelsemine in PSNL mice, mechanical allodynia and thermal hyperalgesia were tested before (0) and 1, 2, 4, and $6 \mathrm{~h}$ after gelsemine was administered ip. To study the dose-dependent anti-hyperalgesic and anti-allodynic effects of gelsemine in a neuropathic pain-like condition, increasing doses of gelsemine $(1,2$, and 4 
$\mathrm{mg} / \mathrm{kg}$ ) were administered $1 \mathrm{~h}$ before mechanical allodynia and thermal hyperalgesia. In addition, mice were injected ip with $4 \mathrm{mg} / \mathrm{kg}$ gelsemine at 8:00 PM to estimate its antinociceptive effects during the active stage of PSNL mice.

To study the effects of gelsemine on sleep disturbance in a neuropathic pain-like condition, gelsemine was administered ip to PSNL mice at 6:30 AM on the experimental day at doses of 1,2 , and $4 \mathrm{mg} / \mathrm{kg}$. In addition, $4 \mathrm{mg} / \mathrm{kg}$ gelsemine was administered ip at 8:30 PM to estimate the possible hypnotic effects of gelsemine during the active stage in normal mice and PSNL mice.

To evaluate any possible non-specific muscle-relaxant or sedative effects of gelsemine, the mean time (s) mice remained on the rota-rod was measured $1,2,4$, and $6 \mathrm{~h}$ after gelsemine $(2,4$, or $8 \mathrm{mg} / \mathrm{kg})$, pregabalin $(100 \mathrm{mg} / \mathrm{kg})$, or vehicle was administered intraperitoneally. The dose of gelsemine that elicited significant antinociceptive effects and promoted sleep was selected, and pregabalin $(100 \mathrm{mg} / \mathrm{kg})$ was used as the positive control $^{[39]}$.

\section{Statistical analysis}

All data were expressed as mean \pm SEM. The time courses of the hourly amounts measured at each stage in PSNL mice treated with gelsemine or vehicle were compared using twoway ANOVA followed by Bonferroni multiple comparisons post hoc test. To assess the total numbers of each vigilance stage during the $3 \mathrm{~h}$ immediately after gelsemine treatment, one-way ANOVA followed by Bonferroni's test was used. The total amount of sleep and wakefulness, the number of stage transitions, the mean duration and the number of bouts of sleep and wakefulness in mice treated with gelsemine and vehicle were assessed using two-way ANOVA followed by the Bonferroni multiple comparisons post hoc test. For multifactorial ANOVA, the treatment (vehicle or drug administration) was evaluated as a between-group factor. For the number of c-Fos immunoreactive neurons, one-way ANOVA followed by the Bonferroni test was used. A $P$-value $<0.05$ was considered to be statistically significant.

\section{Results}

Gelsemine decreased mechanical allodynia and thermal hyperalgesia in PSNL mice

Compared with the sham-operated (sham) group, the mechanical threshold and thermal latency were decreased by $57 \%$ $(P<0.01$, data not shown) and 35\% $(P<0.01$, data not shown) in PSNL mice, respectively, suggesting that PSNL induced tactile allodynia and thermal hyperalgesia in mice. This persistent painful state was reported previously to last for at least $35 \mathrm{~d}$ after PSNL ${ }^{[4]}$.

To test whether gelsemine exerted analgesic effects on neuropathic pain, mechanical threshold and thermal latency were measured after the intraperitoneal administration of gelsemine. As shown in Figure 2A and 2B, gelsemine increased the mechanical threshold significantly for $4 \mathrm{~h}$ and prolonged the thermal latency for $3 \mathrm{~h}$ in PSNL mice. Compared with vehicle control, 1, 2, or $4 \mathrm{mg} / \mathrm{kg}$ gelsemine increased the mechanical threshold to 1.3-, 2.0-, and 2.2-fold (Figure 2C) and the thermal latency to 1.2-, 1.5-, and 1.6-fold, respectively (Figure 2D)
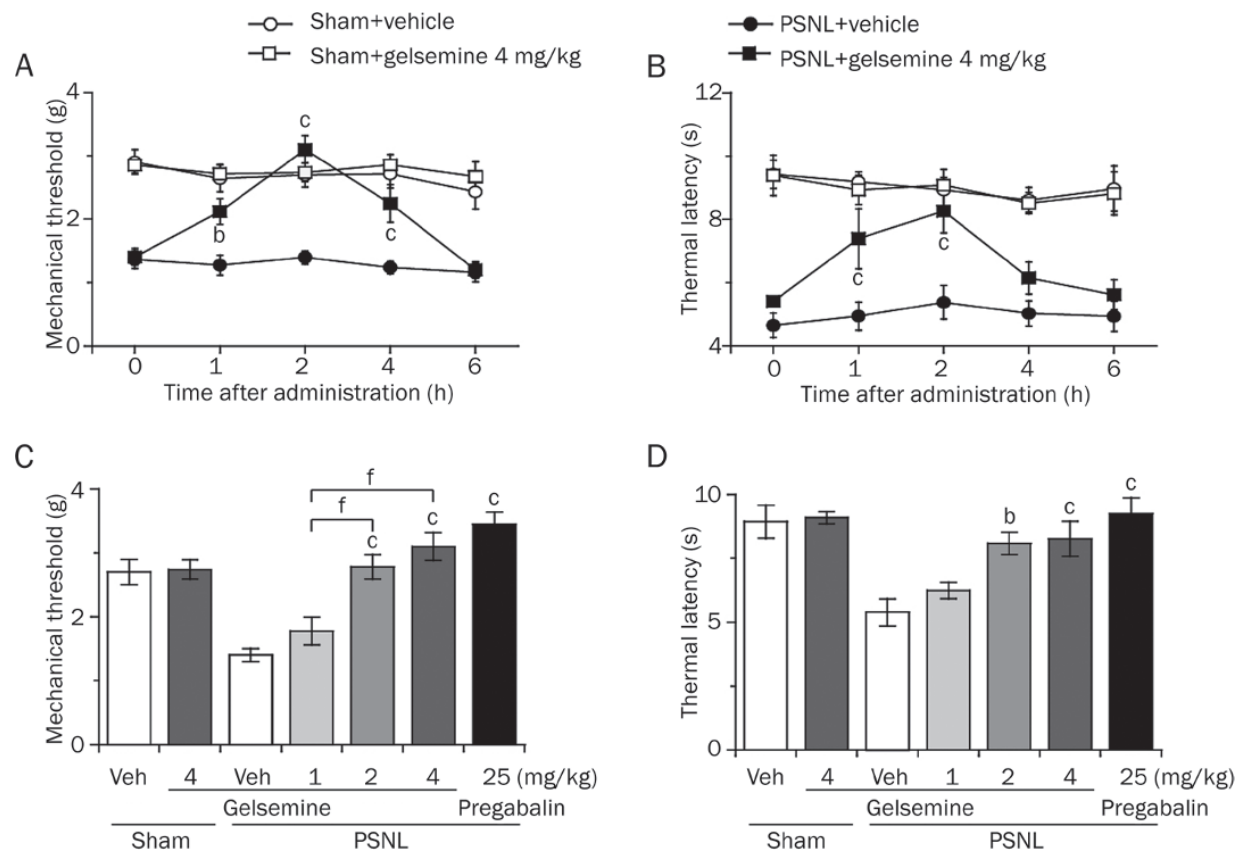

Figure 2. Effects of gelsemine on mechanical threshold and thermal latency. A time course of the effects of gelsemine $(4 \mathrm{mg} / \mathrm{kg})$ on mechanical allodynia (A) and thermal hyperalgesia (B) in sham and PSNL mice. Dose-response effects of gelsemine on mechanical threshold (C) and thermal latency (D) in PSNL mice. Pregabalin was used as a reference drug. Data are expressed as mean \pm SEM $(n=7-8) .{ }^{b} P<0.05$, ${ }^{\mathrm{C}} P<0.01$ vs vehicle-treated PSNL mice. ${ }^{f} P<0.01$ indicates significant differences among doses of gelsemine. 
in PSNL mice $2 \mathrm{~h}$ after intraperitoneal administration. The antinociceptive effects of $4-\mathrm{mg} / \mathrm{kg}$ gelsemine were similar to the effects of $25-\mathrm{mg} / \mathrm{kg}$ pregabalin (the positive control). Furthermore, gelsemine did not affect the mechanical threshold and thermal latency in the sham group (Figure 2C and 2D). These results suggest that gelsemine exerts analgesic effects on PSNL-induced mechanical allodynia and thermal hyperalgesia.

Gelsemine increased NREM sleep and decreased wakefulness in PSNL mice during the light phase

We next investigated whether gelsemine exerted a hypnotic effect in the neuropathic pain-like state during light phase. Different doses of gelsemine $(1,2$, or $4 \mathrm{mg} / \mathrm{kg}$ ) or $25-\mathrm{mg} / \mathrm{kg}$ pregabalin were administered to PSNL mice at 6:30 AM on the experimental day. As shown in Figure 3A, analysis of the time-dependent effects revealed that $4 \mathrm{mg} / \mathrm{kg}$ gelsemine significantly increased NREM sleep in PSNL mice for $3 \mathrm{~h}$ compared with vehicle control, with a maximal increase of 0.7 -fold in the first hour; however, REM sleep remained unchanged.

Figure 3B summarizes the total amount of time spent in NREM sleep, REM sleep, and wakefulness in PSNL mice during the first $3 \mathrm{~h}$ after treatment with vehicle or different doses of gelsemine. Compared with the vehicle control, 1, 2, or 4 $\mathrm{mg} / \mathrm{kg}$ gelsemine increased NREM sleep to 1.2-( $P>0.05), 1.4-$ $(P<0.01)$, and 1.5 -fold $(P<0.01)$ and decreased wakefulness by 23\% $(P<0.05), 40 \%(P<0.01)$, and 48\% $(P<0.01)$, respectively. However, the amount of REM sleep remained unchanged. Similarly, pretreatment with $25 \mathrm{mg} / \mathrm{kg}$ pregabalin increased the total amount of NREM sleep to 1.4 -fold $(P<0.01)$ and decreased wakefulness by $39 \%(P<0.01)$ compared with vehicle control during the same $3-\mathrm{h}$ period. However, $4 \mathrm{mg} / \mathrm{kg}$ gelsemine did not change the amount of NREM sleep, REM sleep, and wakefulness in the sham mice (data not shown). These findings clearly indicate that gelsemine increased NREM sleep in PSNL but not sham mice.

\section{Gelsemine changed the sleep architecture in PSNL mice}

To investigate the alterations in sleep architecture after gelsemine administration, we measured the total episode numbers, mean durations, the numbers of NREM and REM sleep bouts, and EEG power density.

During the first $3 \mathrm{~h}$ after dosing in the light period, $4 \mathrm{mg} / \mathrm{kg}$ gelsemine increased the total number of episodes of NREM sleep in PSNL mice to 1.3 -fold $(P<0.05$; Figure $4 \mathrm{~A})$, and decreased the mean duration of wakefulness significantly by $53.6 \%(P<0.01$; Figure $4 \mathrm{~B})$. In contrast, the mean duration of NREM and REM sleep remained unchanged compared with vehicle control. Figure $4 \mathrm{C}$ and $4 \mathrm{D}$ show that there were no significant changes in the distribution of bouts of NREM and REM sleep.

Next, we determined the EEG power spectra of NREM sleep in PSNL mice. The power of each $0.5-\mathrm{Hz}$ bin was first averaged across the sleep stages individually, and then normalized by calculating the percentage of each bin from the total power $(0-24.5 \mathrm{~Hz})$ of the individual animal. As shown in Figure 4E, there were no significant differences in the EEG power density of NREM sleep between the gelsemine- and vehicle control-
A
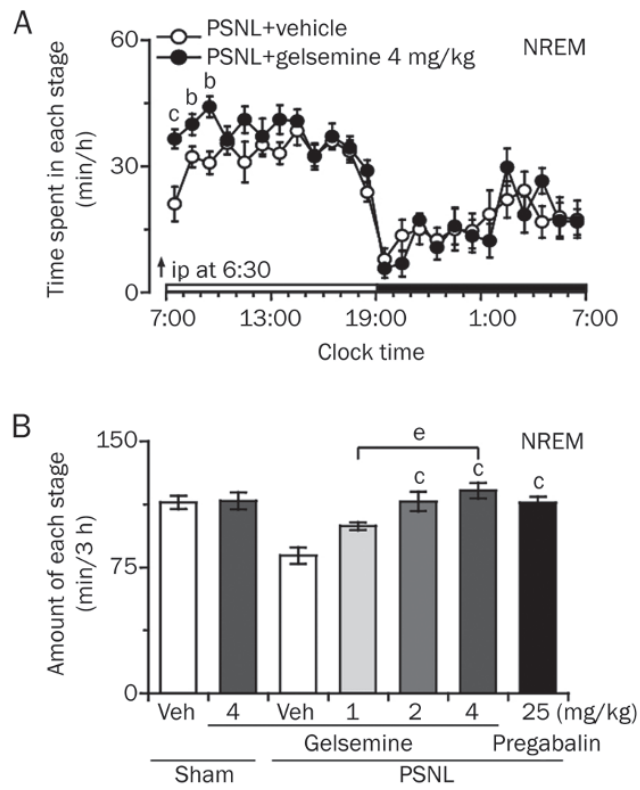
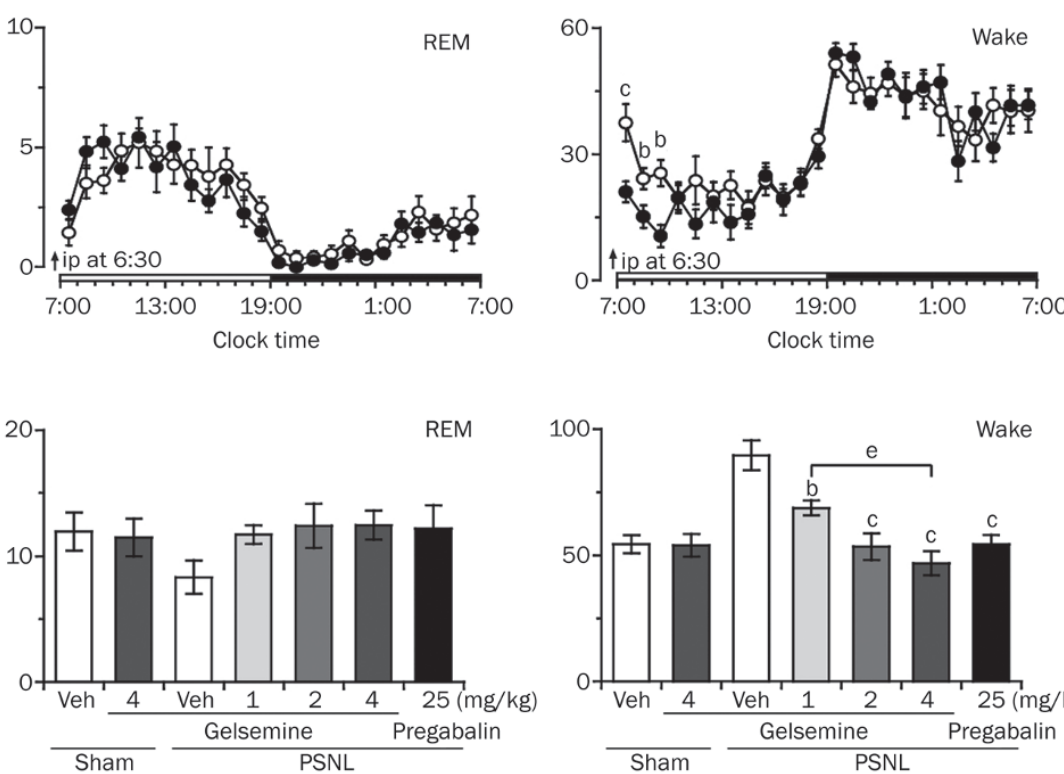

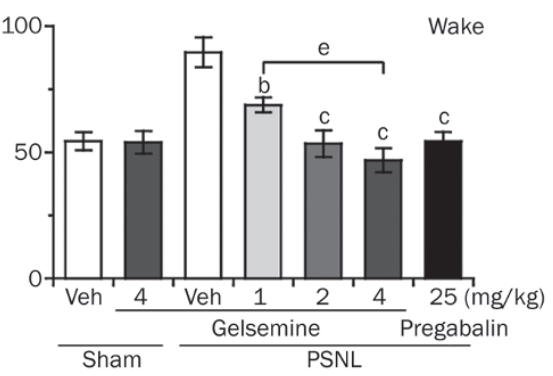

Figure 3. Sleep-stage distributions produced by the intraperitoneal administration of gelsemine in PSNL mice. (A) Time course of the changes in NREM sleep, REM sleep, and wakefulness in PSNL mice treated with gelsemine. Each circle represents the hourly mean amount of each stage. The horizontal open and filled bars on the X-axes indicate the $12 \mathrm{~h}$ light and $12 \mathrm{~h}$ dark periods, respectively. Data are expressed as mean \pm SEM $(n=8)$. ${ }^{b} P<0.05$, ${ }^{c} P<0.01$ vs vehicle control group. (B) Dose-dependent effect of gelsemine on the total time spent in NREM sleep, REM sleep, and wakefulness for $3 \mathrm{~h}$ after administration in PSNL mice. Data are expressed as mean \pm SEM $(n=8) .{ }^{b} P<0.05,{ }^{c} P<0.01$ vs vehicle-treated PSNL mice; ${ }^{\mathrm{e}} P<0.05$ indicates significant differences among the gelsemine doses. 
treated groups. Collectively, these data suggest that gelsemine increased the total number of episodes of NREM sleep, but did not change the EEG power spectra in PSNL mice.

No hypnotic effects of gelsemine given in the dark phase in normal mice and PSNL mice
To determine the hypnotic effects of gelsemine in the dark phase when mice spend most time in wakefulness, $4 \mathrm{mg} / \mathrm{kg}$ gelsemine was administered ip to mice at 8:30 PM. The results showed that gelsemine did not affect the sleep-wake profiles (Figure 5A). We next calculated the total amount of time spent in NREM sleep, REM sleep, and wakefulness during the $3 \mathrm{~h}$
A
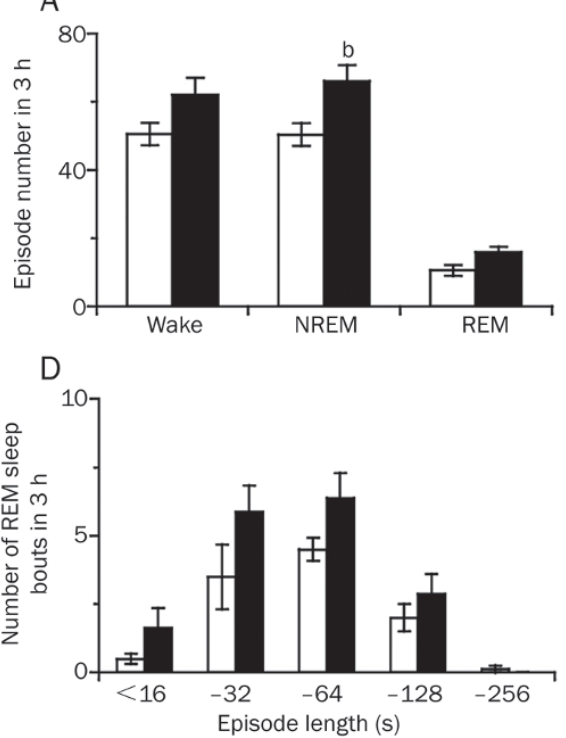

B

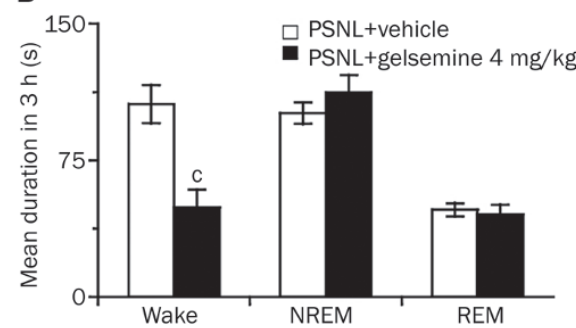

E

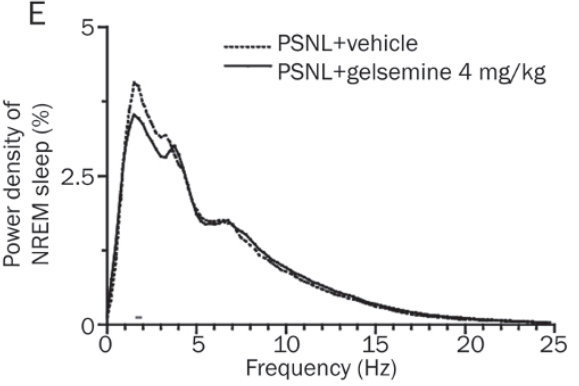

C

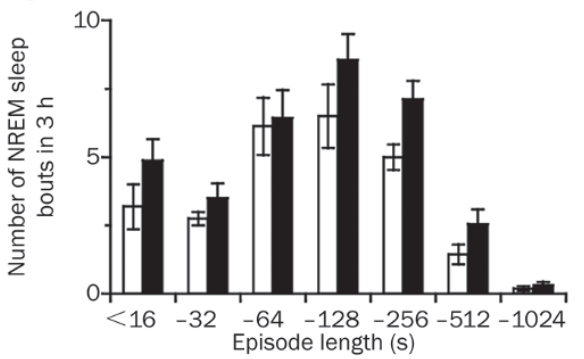

Figure 4. Characteristics of sleep-wake episodes and mean durations during the $3 \mathrm{~h}$ following gelsemine administration. (A) Total episode numbers, (B) mean durations, ( $C$ and $D$ ) the numbers of sleep bouts, and $(E)$ the EEG power density of NREM sleep. Open and filled bars show the profiles of the vehicle- and gelsemine-treated mice, respectively. Data are presented as mean \pm SEM $(n=8)$. ${ }^{b} P<0.05,{ }^{c} P<0.01$ vs vehicle-treated PSNL mice.

A
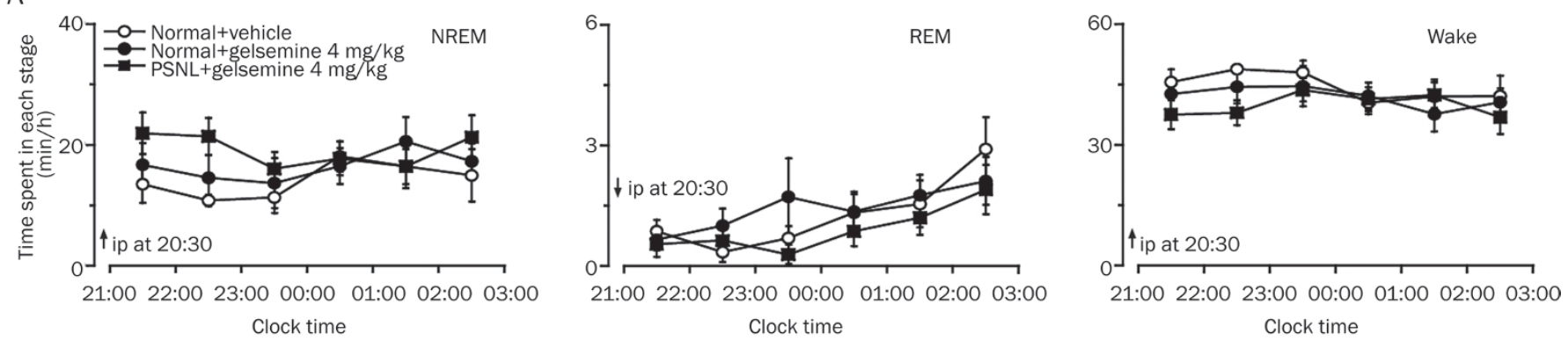

B
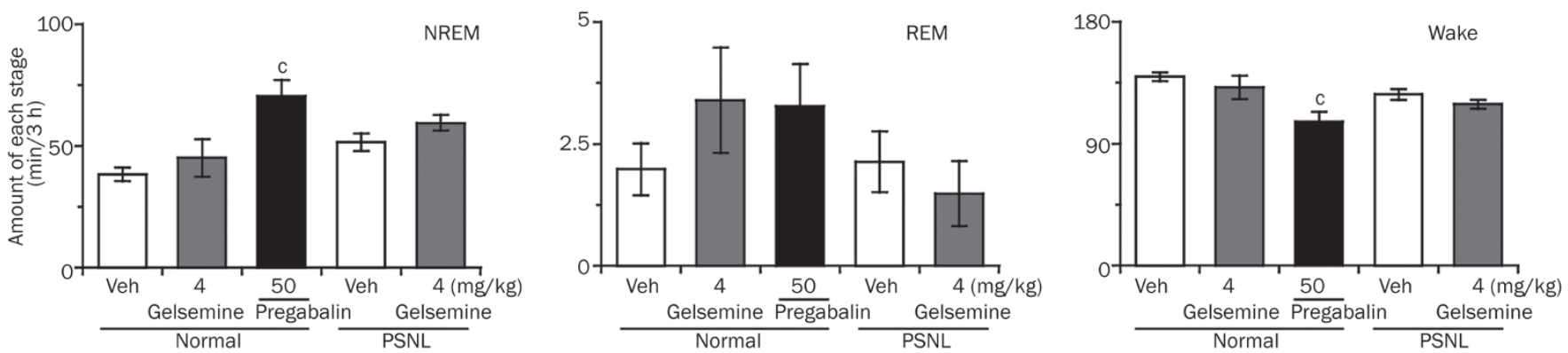

Figure 5. Sleep-stage distributions in normal mice and PSNL mice after the intraperitoneal administration of gelsemine. (A) Changes in the time course in NREM sleep, REM sleep, and wakefulness after the ip administration of $4 \mathrm{mg} / \mathrm{kg}$ gelsemine to normal mice and PSNL mice. Each data dot represents the hourly mean time spent in each stage. (B) Effect of gelsemine on the total time spent in NREM sleep, REM sleep, and wakefulness for $3 \mathrm{~h}$ after administration to normal mice and PSNL mice. Data are expressed as mean \pm SEM $(n=6-7) .{ }^{c} P<0.01$ vs vehicle-treated normal mice. 
following gelsemine treatment. There was no difference in the sleep-wake cycles between gelsemine treatment and vehicle control in either normal or PSNL mice (Figure 5B).

Gelsemine decreased mechanical allodynia in PSNL mice in the dark phase

We next investigated whether gelsemine exerted analgesic effects in the neuropathic pain-like state during the dark phase. The mechanical threshold was measured $1.5 \mathrm{~h}$ after the ip administration of gelsemine at 8:00 PM. Gelsemine $(4 \mathrm{mg} / \mathrm{kg})$ significantly increased the mechanical threshold of PSNL mice to 1.8 -fold $(P<0.01)$. In contrast, gelsemine did not affect the mechanical threshold in the sham group (Figure 6).

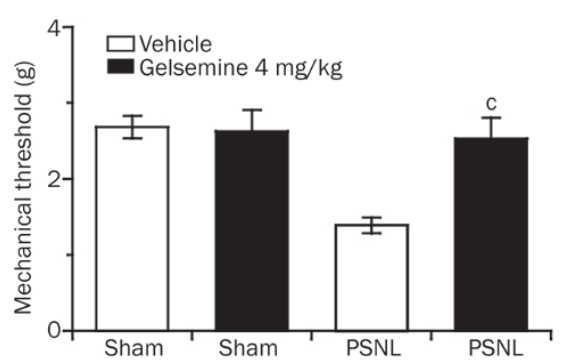

Figure 6. Effects of gelsemine on the mechanical threshold in the active stage. Mice were treated with vehicle or gelsemine at 8:00 PM prior to starting threshold evaluations. Data are expressed as mean \pm SEM $(n=8-11)$. ${ }^{c} P<0.01$ vs vehicle-treated PSNL mice.

Gelsemine did not impair the motor coordination of the PSNL mice in the rota-rod test

To eliminate the possibility that motor impairment accounted for the analgesic effects of gelsemine, we evaluated the motor performance of sham and PSNL mice following the administration of gelsemine. As shown in Figure 7, there was no difference in the rota-rod staying time between sham or PSNL mice injected with vehicle. Gelsemine $(4 \mathrm{mg} / \mathrm{kg})$ elicited significant antinociceptive effects, but did not alter motor performance compared with vehicle control. All animals treated with $4 \mathrm{mg} / \mathrm{kg}$ gelsemine remained on the rota-rod until the cut-off latency of $180 \mathrm{~s}$. However, $100 \mathrm{mg} / \mathrm{kg}$ pregabalin significantly reduced the rota-rod staying time by $37 \%(P<0.01)$ and $50 \%(P<0.01) 1 \mathrm{~h}$ and $2 \mathrm{~h}$ after administration to PSNL mice, respectively, compared with vehicle control. This suggests that the antinociceptive effects of gelsemine observed in PSNL mice did not impair motor function, confirming that gelsemine induces an antinociceptive effect.

Effects of gelsemine on c-Fos expression in the ACC of PSNL and sham mice

To further test our hypothesis that the ACC might be involved in the mechanism by which gelsemine improves sleep quality in patients with disorders caused by neuropathic pain, we measured the mechanical pain thresholds $1.5 \mathrm{~h}$ after drug treatment, and then immediately sampled the brain to count the number of
A

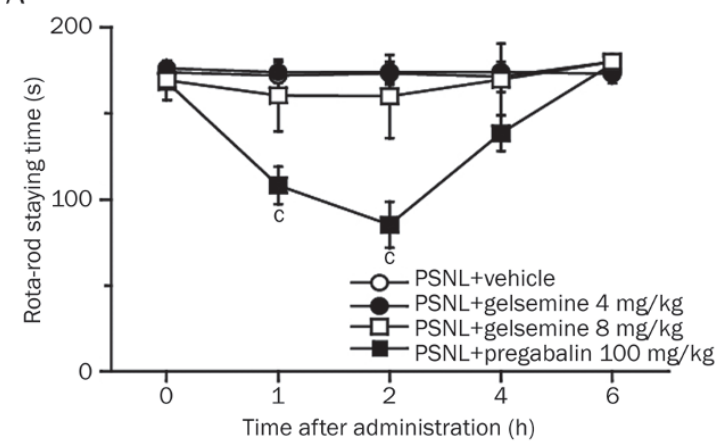

B

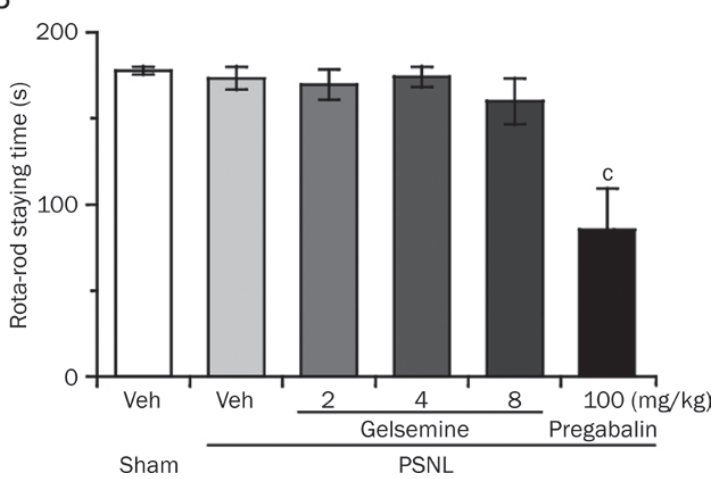

Figure 7. Effect of gelsemine on the motor performance of mice in the rota-rod test. (A) Time course of the effects of gelsemine and pregabalin on the rota-rod staying time of PSNL mice. (B) Dose-dependent effects of gelsemine on the rota-rod staying time of PSNL mice. The mean time (s) mice remained on the rota-rod was measured $2 \mathrm{~h}$ after the ip administration of gelsemine, pregabalin (reference drug) or vehicle. Data are expressed as mean \pm SEM $(n=6-8)$. ${ }^{c} P<0.01$ vs vehicle-treated PSNL mice.

c-Fos-positive neurons in the ACC of PSNL and sham mice. In Figure $8 \mathrm{~A}$ and $8 \mathrm{~B}$, the site of the ACC was verified according to the coordinates provided by Franklin and Paxinoson on the left. Representative photomicrographs of c-Fos immunostaining in the ACC of mice treated with $4 \mathrm{mg} / \mathrm{kg}$ gelsemine or vehicle are shown in Figure 8C-8F.

As shown in Figure $8 \mathrm{G}$, gelsemine $(4 \mathrm{mg} / \mathrm{kg}$ ) increased the mechanical threshold in the PSNL group significantly after administration, but had no effect on sham mice compared with the control group. These findings suggest that gelsemine exerted an analgesic effect in the PSNL group. Analysis of the number of c-Fos immunoreactive cnuclei demonstrated that PSNL increased c-Fos expression in the ACC to 3.2-fold compared with the sham group (Figure $8 \mathrm{H}$ ). In addition, $4 \mathrm{mg} / \mathrm{kg}$ gelsemine decreased c-Fos expression in the ACC of PSNL mice significantly by $58 \%(P<0.01)$, but had no effect in sham mice. These data suggest that the ACC might be responsible for the hypnotic or analgesic effects of gelsemine in PSNL mice.

\section{Discussion}

The present study demonstrated that $4 \mathrm{mg} / \mathrm{kg}$ gelsemine exerted antinociceptive effects and improved sleep distur- 

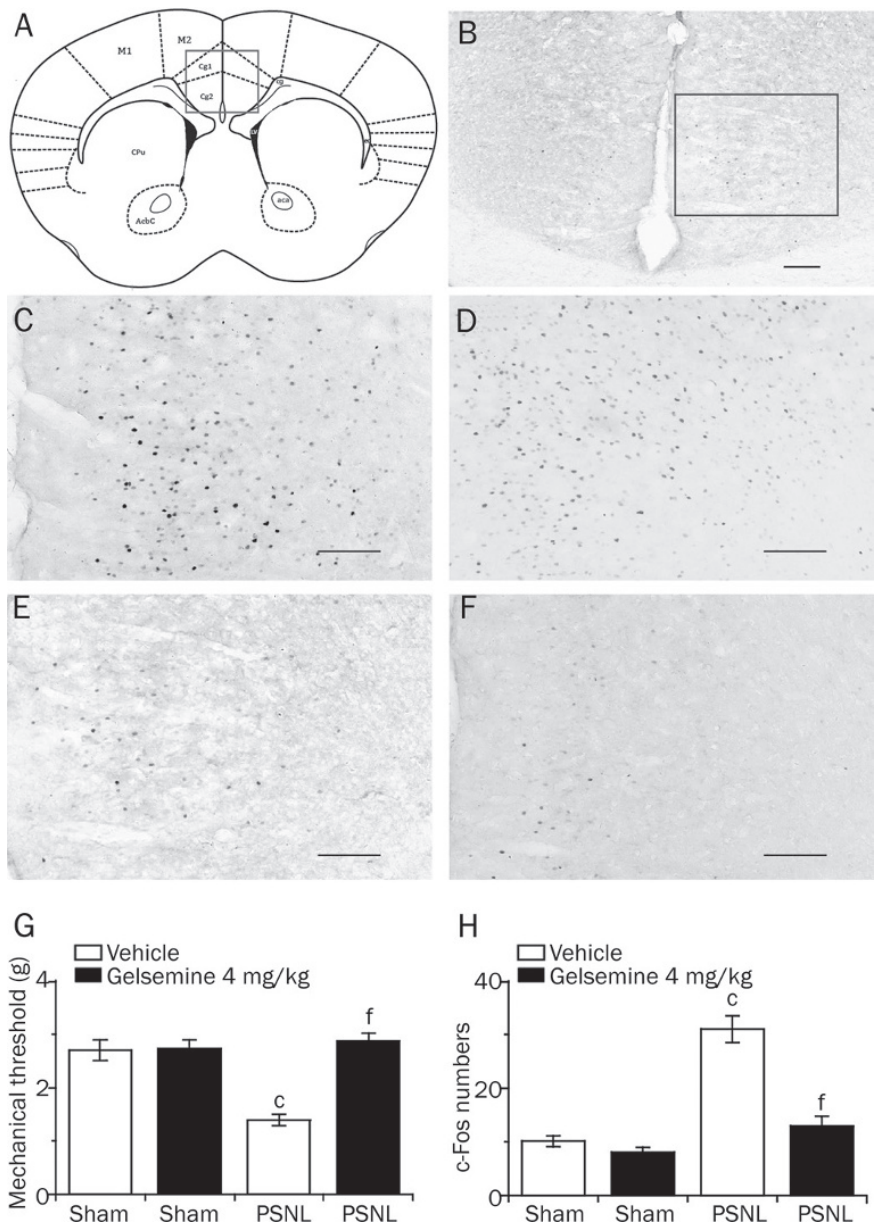

Figure 8. Effects of gelsemine on c-Fos expression in the ACC of PSNL and sham mice. (A and B) The site of the ACC was verified according to the coordinates provided by Franklin and Paxinos shown on the left. (C-F) Representative photomicrographs of c-Fos expression in the ACC of the $\mathrm{PSNL}+4 \mathrm{mg} / \mathrm{kg}$ gelsemine group (C), the PSNL+vehicle group (D), the sham $+4 \mathrm{mg} / \mathrm{kg}$ gelsemine group $(\mathrm{E})$, and the sham+vehicle group $(\mathrm{F})$; scale bars $=100 \mu \mathrm{m}$. (G) Mechanical allodynia was measured $90 \mathrm{~min}$ after treatment with vehicle or gelsemine in PSNL and sham mice. $(H)$ Number of c-Fos-expressing cells in the ACC of PSNL and sham mice 120 min after treatment with vehicle or gelsemine. Data are expressed as mean \pm SEM $(n=8) .{ }^{\mathrm{c}} P<0.01$ vs vehicle-treated sham mice; ${ }^{f} P<0.01$ vs vehicle-treated PSNL mice.

bance during the light phase in PSNL mice. The mean duration of wakefulness was shortened significantly and the total number of episodes of NREM sleep was increased after gelsemine administration during the light period, suggesting that gelsemine might exert a hypnotic function by reducing long lasting continuous wakefulness in mice with neuropathic pain. We first showed that the ip administration of gelsemine exerted antinociceptive effects in PSNL mice at both the mechanical and thermal pain thresholds, and that this effect lasted for $\sim 4 \mathrm{~h}$ after administration. Recent studies reported that gelsemine produced a specific antinociceptive effect after intrathecal administration via actions on spinal a 3 glycine receptors ${ }^{[18]}$. The current data demonstrated that the intraperi- toneal injection of gelsemine also elicited effective pain relief. Gelsemine did not change the mechanical threshold and thermal latency in sham mice, suggesting that the analgesic effects of gelsemine were potent under specific pathological conditions. Moreover, $4 \mathrm{mg} / \mathrm{kg}$ gelsemine, which elicited antinociceptive effects, did not alter motor performance, suggesting that the analgesic effects of $4 \mathrm{mg} / \mathrm{kg}$ gelsemine were not the result of a motor side effect. Taken together, these data support the effectiveness of gelsemine for the treatment of neuropathic pain, which is one of the most common and difficult types of chronic pain.

The PSNL mice exhibited poor sleep quality with disrupted sleep during the light phase from $d 7$ after surgery. These results are consistent with previous reports whereby chronic neuropathic pain caused a significant increase in wakefulness and a decrease in NREM sleep at $\mathrm{d} 7$, and persisted for at least $28 \mathrm{~d}$ after PSNL ${ }^{[4,21,22]}$. Sleep disturbance, one of the most common results of pain, confounds the treatment of neuropathic pain significantly ${ }^{[6,24,40-42]}$. Therefore, ideal pain management strategies should be designed to treat the whole patient, not just the pain. Desipramine as a tricyclic antidepressant that treats neuropathic pain and alleviates pain in diabetic peripheral neuropathy patients, but is known to disrupt sleep continuity $^{\left[{ }^{[33]}\right.}$. In addition, paroxetine and duloxetine have been used to treat patients with neuropathic pain ${ }^{[4,45]}$. However, they increased wakefulness and suppressed sleep ${ }^{[46]}$. The current study revealed that $4 \mathrm{mg} / \mathrm{kg}$ gelsemine obviously improved sleep disturbance during the light phase (the "sleep period" for mice) in PSNL mice. Both the total time and the number of bouts of NREM sleep in PSNL mice were increased significantly during the first $3 \mathrm{~h}$ after gelsemine administration. In addition, the relative mean duration and total time of wakefulness decreased. These data suggest that gelsemine likely induced more sleep onsets in the PSNL mice, and thereby decreased the long-lasting wakefulness stage during the light phase. Interestingly, the current data also showed that gelsemine exerted antinociceptive effects in PSNL mice during the dark phase, but had no hypnotic effects in PSNL mice during the dark phase. Thus, we concluded that the hypnotic effects of gelsemine were exerted via a different mechanism than its antinociceptive effect via the spinal a 3 glycine receptors.

To further investigate the mechanism of the antinociceptive and hypnotic effects of gelsemine, we measured activation of the ACC, a brain cortical region that is related to both pain and sleep. A previous study showed that neuropathic pain might decrease the release of GABA at the synaptic cleft of the ACC region in PSNL mice ${ }^{[21]}$. Interestingly, the present study revealed that PSNL increased c-Fos expression in ACC neurons significantly, whereas $4 \mathrm{mg} / \mathrm{kg}$ gelsemine decreased the expression of c-Fos in the ACC of PSNL mice remarkably. However, these effects were not observed in the sham mice. Previous studies demonstrated that the ACC is activated during pain conditions ${ }^{[47,48]}$, which is consistent with the current study. The ACC might be the central site of the antinociceptive and hypnotic effects of gelsemine. Moreover, the current 
results regarding c-Fos expression in the ACC might further explain why gelsemine had no hypnotic effects during the dark phase, since the ACC is not activated during the "wake phase" of mice ${ }^{[49,50]}$.

Collectively, these results provide evidence that treatment with gelsemine could be an effective method for treating neuropathic pain and improving sleep disturbance in neuropathic pain patients without negatively affecting their daily life.

\section{Conclusion}

In conclusion, this study demonstrated that gelsemine exerted antinociceptive effects and increased NREM sleep for $3 \mathrm{~h}$ after its intraperitoneal administration in a chronic pain insomnia model. Based on the c-Fos findings in the ACC, these effects could be attributed to inhibition of the ACC hyperactive neurons. Therefore, we conclude that gelsemine could be a promising efficacious treatment for neuropathic pain, as well as sleep disturbance.

\section{Abbreviations}

ACC, anterior cingulate cortex; EEG, electroencephalogram; EMG, electromyogram; NREM, non-rapid eye movement; PSNL, partial sciatic nerve ligation; REM, rapid eye movement.

\section{Acknowledgements}

This study was supported in part by grants-in-aid for scientific research from the the National Basic Research Program of China (2011CB711000 and 2015CB856401), the National Natural Science Foundation of China (31171010, 31121061, 31271164, 31471064, 81420108015, J1210041, and 81301135), Shanghai Committee of Science and Technology (13140903100, 14JC1400900, 13dz2260700 and 13ZR1403200), a key laboratory program of the Education Commission of Shanghai Municipality (ZDSYS14005), and the Shanghai Leading Academic Discipline Project (B119).

\section{Author contribution}

Yu-er WU and Ya-dong LI designed the study, conducted the experiments and drafted the manuscript; Yan-jia LUO and Tian-xiao WANG coordinated the experiments; Huijing WANG was involved in data analysis, discussion of the experiments and draft of the manuscript; Shuo-nan CHEN was involved in the animal experiments; Wei-min QU was involved in the discussion of the experiments and data analysis; Zhi-li HUANG was involved in discussion of the experiments and drafts of the manuscript. All authors read and approved the final manuscript.

\section{References}

1 Jensen TS, Finnerup NB. Neuropathic pain treatment: a further step forward. Lancet 2009; 374: 1218-9.

2 Treede RD, Jensen TS, Campbell JN, Cruccu G, Dostrovsky JO, Griffin JW, et al. Neuropathic pain: redefinition and a grading system for clinical and research purposes. Neurology 2008; 70: 1630-5.

3 Narita M, Niikura K, Nanjo-Niikura K, Furuya M, Yamashita A, Saeki $M$, et al. Sleep disturbances in a neuropathic pain-like condition in the mouse are associated with altered GABAergic transmission in the cingulate cortex. Pain 2011; 152: 1358-72.

4 Liu YY, Yin D, Chen L, Qu WM, Chen CR, Laudon M, et al. Piromelatine exerts antinociceptive effect via melatonin, opioid, and $5 \mathrm{HT}$ receptors and hypnotic effect via melatonin receptors in a mouse model of neuropathic pain. Psychopharmacology (Berl) 2014; 231: 3973-85.

5 Morin CM, Gibson D, Wade J. Self-reported sleep and mood disturbance in chronic pain patients. Clin J Pain 1998; 14: 311-4.

6 Smith MT, Haythornthwaite JA. How do sleep disturbance and chronic pain inter-relate? Insights from the longitudinal and cognitivebehavioral clinical trials literature. Sleep Med Rev 2004; 8: 119-32.

7 Pilowsky I, Crettenden I, Townley M. Sleep disturbance in pain clinic patients. Pain 1985; 23: 27-33.

8 Peles E, Schreiber S, Adelson M. Documented poor sleep among methadone-maintained patients is associated with chronic pain and benzodiazepine abuse, but not with methadone dose. Eur Neuropsychopharmacol 2009; 19: 581-8.

9 Argoff CE. The coexistence of neuropathic pain, sleep, and psychiatric disorders - A novel treatment approach. Clin J Pain 2007; 23: 15-22.

10 Dutt V, Thakur S, Dhar VJ, Sharma A. The genus Gelsemium: An update. Pharmacogn Rev 2010; 4: 185-94.

11 Dutt V, Dhar VJ, Sharma A. Antianxiety activity of Gelsemium sempervirens. Pharm Biol 2010; 48: 1091-6.

12 Xu Y, Qiu HQ, Liu H, Liu M, Huang ZY, Yang J, et al. Effects of koumine, an alkaloid of Gelsemium elegans Benth, on inflammatory and neuropathic pain models and possible mechanism with allopregnanolone. Pharmacol Biochem Behav 2012; 101: 504-14.

13 Rujjanawate C, Kanjanapothi D, Panthong A. Pharmacological effect and toxicity of alkaloids from Gelsemium elegans Benth. J Ethnopharmacol 2003; 89: 91-5.

14 Liu M, Huang HH, Yang J, Su YP, Lin HW, Lin LQ, et al. The active alkaloids of Gelsemium elegans Benth are potent anxiolytics. Psychopharmacology (Berl) 2013; 225: 839-51.

15 Meyer L, Boujedaini N, Patte-Mensah C, Mensah-Nyagan AG. Pharmacological effect of gelsemine on anxiety-like behavior in rat. Behav Brain Res 2013; 253: 90-4.

16 Jin GL, Su YP, Liu M, Xu Y, Yang J, Liao KJ, et al. Medicinal plants of the genus Gelsemium (Gelsemiaceae, Gentianales)-A review of their phytochemistry, pharmacology, toxicology and traditional use. J Ethnopharmacol 2014; 152: 33-52.

17 Bellavite P, Magnani P, Marzotto M, Conforti A. Assays of homeopathic remedies in rodent behavioural and psychopathological models. Homeopathy 2009; 98: 208-27.

18 Zhang JY, Gong N, Huang JL, Guo LC, Wang YX. Gelsemine, a principal alkaloid from Gelsemium sempervirens Ait, exhibits potent and specific antinociception in chronic pain by acting at spinal alpha3 glycine receptors. Pain 2013; 154: 2452-62.

19 Johansen JP, Fields HL. Glutamatergic activation of anterior cingulate cortex produces an aversive teaching signal. Nat Neurosci 2004; 7 : 398-403.

20 Zhuo M. Cortical excitation and chronic pain. Trends Neurosci 2008; 31: 199-207.

21 Narita M, Niikura K, Nanjo-Niikura K, Narita M, Furuya M, Yamashita $A$, et al. Sleep disturbances in a neuropathic pain-like condition in the mouse are associated with altered GABAergic transmission in the cingulate cortex. Pain 2011; 152: 1358-72.

22 Takemura Y, Yamashita A, Horiuchi H, Furuya M, Yanase M, Niikura K, et al. Effects of gabapentin on brain hyperactivity related to pain and sleep disturbance under a neuropathic pain-like state using fMRI and brain wave analysis. Synapse 2011; 65: 668-76.

23 Zhao X, Xu Y, Zhao Q, Chen CR, Liu AM, Huang ZL. Curcumin exerts 
antinociceptive effects in a mouse model of neuropathic pain: descending monoamine system and opioid receptors are differentially involved. Neuropharmacology 2012; 62: 843-54.

24 Dixon WJ. Efficient analysis of experimental observations. Annu Rev Pharmacol Toxicol 1980; 20: 441-62.

25 Willemen HL, Eijkelkamp N, Wang H, Dantzer R, Dorn GW 2nd, Kelley $\mathrm{KW}$, et al. Microglial/macrophage GRK2 determines duration of peripheral IL-1beta-induced hyperalgesia: contribution of spinal cord CX3CR1, p38 and IL-1 signaling. Pain 2010; 150: 550-60.

26 Wang HJ, Heijnen CJ, Eijkelkamp N, Garza Carbajal A, Schedlowski $\mathrm{M}$, Kelley KW, et al. GRK2 in sensory neurons regulates epinephrineinduced signalling and duration of mechanical hyperalgesia. Pain 2011; 152: 1649-58.

27 Wang HJ, Heijnen CJ, van Velthoven CTJ, Willemen HLDM, Ishikawa $\mathrm{Y}$, Zhang $\mathrm{X}$, et al. Balancing GRK2 and EPAC1 levels prevents and relieves chronic pain. J Clin Invest 2013; 123: 5023-34.

28 Chaplan SR, Bach FW, Pogrel JW, Chung JM, Yaksh TL. Quantitative assessment of tactile allodynia in the rat paw. J Neurosci Methods 1994; 53: 55-63.

29 Hargreaves K, Dubner R, Brown F, Flores C, Joris J. A new and sensitive method for measuring thermal nociception in cutaneous hyperalgesia. Pain 1988; 32: 77-88.

30 Xu Q, Xu XH, Qu WM, Lazarus M, Urade Y, Huang ZL. A mouse model mimicking human first night effect for the evaluation of hypnotics. Pharmacol Biochem Behav 2014; 116: 129-36.

31 Wang YQ, Takata Y, Li R, Zhang Z, Zhang MQ, Urade Y, et al. Doxepin and diphenhydramine increased non-rapid eye movement sleep through blockade of histamine $\mathrm{H} 1$ receptors. Pharmacol Biochem Behav 2015; 129: 56-64.

32 Huang ZL, Mochizuki T, Qu WM, Hong ZY, Watanabe T, Urade Y, et al. Altered sleep-wake characteristics and lack of arousal response to H3 receptor antagonist in histamine $\mathrm{H} 1$ receptor knockout mice. Proc Natl Acad Sci U S A 2006; 103: 4687-92.

33 Qu WM, Huang ZL, Xu XH, Matsumoto N, Urade Y. Dopaminergic D1 and D2 receptors are essential for the arousal effect of modafinil. J Neurosci 2008; 28: 8462-9.

$34 \mathrm{Xu} X \mathrm{XH}$, Qiu MH, Dong H, Qu WM, Urade Y, Huang ZL. GABA transporter-1 inhibitor NO-711 alters the EEG power spectra and enhances non-rapid eye movement sleep during the active phase in mice. Eur Neuropsychopharmacol 2014; 24: 585-94.

35 Yan MM, Xu XH, Huang ZL, Yao MH, Urade Y, Qu WM. Selection of optimal epoch duration in assessment of rodent sleep-wake profiles. Sleep Biol Rhythms 2011; 9: 46-55.

36 Qu WM, Xu XH, Yan MM, Wang YQ, Urade Y, Huang ZL. Essential role of dopamine D2 receptor in the maintenance of wakefulness, but not in homeostatic regulation of sleep, in mice. J Neurosci 2010; 30 : 4382-9.

37 Kuribara H, Higuchi Y, Tadokoro S. Effects of central depressants on rota-rod and traction performances in mice. Jpn J Pharmacol 1977;
27: $117-26$.

38 Chen CR, Tan R, Qu WM, Wu Z, Wang Y, Urade Y, et al. Magnolol, a major bioactive constituent of the bark of Magnolia officinalis, exerts antiepileptic effects via the GABA/benzodiazepine receptor complex in mice. Br J Pharmacol 2011; 164: 1534-46.

39 Wang TX, Yin D, Guo W, Liu YY, Li YD, Qu WM, et al. Antinociceptive and hypnotic activities of pregabalin in a neuropathic pain-like model in mice. Pharmacol Biochem Behav 2015; 135: 31-9.

40 Onen SH, Alloui A, Gross A, Eschaller A, Dubray C. The effects of total sleep deprivation, selective sleep interruption and sleep recovery on pain tolerance thresholds in healthy subjects. J Sleep Res 2001; 10 : 35-42.

41 Fishbain DA. Approaches to treatment decisions for psychiatric comorbidity in the management of the chronic pain patient. Med Clin North Am 1999; 83: 737-60.

42 Bellavite P, Magnani P, Zanolin E, Conforti A. Homeopathic Doses of Gelsemium sempervirens Improve the Behavior of Mice in Response to Novel Environments. Evid Based Complement Alternat Med 2011; 2011: 362517.

43 Sindrup SH, Gram LF, Skjold T, Grodum E, Brosen K, Becknielsen H. Clomipramine vs desipramine vs placebo in the treatment of diabetic neuropathy symptoms. A double-blind cross-over study. Br J Clin Pharmacol 1990; 30: 683-91.

44 Sindrup SH, Gram LF, Brosen K, Eshoj O, Mogensen EF. The selective serotonin reuptake inhibitor paroxetine is effective in the treatment of diabetic neuropathy symptoms. Pain 1990; 42: 135-44.

45 Goldstein DJ, Lu YL, Detke MJ, Lee TC, lyengar S. Duloxetine vs placebo in patients with painful diabetic neuropathy. Pain 2005; 116 : 109-18.

46 Thase ME. Antidepressant treatment of the depressed patient with insomnia. J Clin Psychiatry 1999; 60: 28-31.

47 Casey KL. Forebrain mechanisms of nociception and pain: Analysis through imaging. Proc Natl Acad Sci U S A 1999; 96: 7668-74.

48 Rainville P, Bushnell MC, Duncan GH. Representation of acute and persistent pain in the human CNS: potential implications for chemical intolerance. Ann N Y Acad Sci 2006; 933: 130-41.

49 Horovitz SG, Braun AR, Carr WS, Picchioni D, Balkin TJ, Fukunaga M, et al. Decoupling of the brain's default mode network during deep sleep. Proc Natl Acad Sci U S A 2009; 106: 11376-81.

50 Koike T, Kan S, Misaki M, Miyauchi S. Connectivity pattern changes in default-mode network with deep non-REM and REM sleep. Neurosci Res 2011; 69: 322-30.

(c) This work is licensed under the Creative इOMERIGHSHESERVD Commons Attribution-NonCommercial-No Derivative Works 3.0 Unported License. To view a copy of this license, visit http://creativecommons.org/licenses/ by-nc-nd/3.0/ 